\title{
Hydrodynamic Forces on a Submerged Circular Cylinder in Two-layer Fluid with an Ice-cover
}

\author{
Manomita Sahu and Dilip Das* \\ Department of Mathematics, \\ Diamond Harbour Women's University, \\ Sarisha, West Bengal-743368, India
}

${ }^{*}$ Correspondng author: e-mail : dilipdas99@gmail.com, tel: 919433567457

\begin{abstract}
We consider problems based on linear water wave theory concerning the interaction of wave with horizontal circular cylinder submerged in two-layer ocean consisting of a upper layer of finite depth bounded above by an ice-cover and below by an infinite layer of fluid of greater density, the ice-cover being modelled as an elastic plate of very small thickness. Using the method of multipoles, we formulate the problems of hydrodynamic forces on a submerged cylinder in either the upper or the lower layer. The vertical and horizontal forces on the circular cylinder are obtained and depicted graphically against the wave number for various values of flexural rigidity of ice-cover to show the effect of the presence of ice-cover on these quantities. Also when the flexural rigidity and surface density of the ice-cover are taken to be zero, the ice-cover tends to a free-surface. Then all the forces are the same as in the case of two-layer fluid with free surface.
\end{abstract}

Keywords: Two-layer fluid, Water wave scattering, Submerged circular cylinder, Icecover, Vertical and horizontal forces.

\section{Introduction}

The problem of scattering and radiation problems for a submerged as well as floating bodies in water have been explored for decades for various shapes of bodies. Long back Dean [1], Ursell [2] investigated the problem of water wave scattering by a horizontal circular cylinder fully submerged in deep water using the method of multipoles. This method also used in various field of theoretical physics (cf. Jackson [3], Morse and Feshbach [4]). Evans and Linton [5] considered both the scattering and radiation water wave problems by a submerged circular cylinder in finite depth water as part of a need to determine accurately the natural frequencies of oscillation of a highly buoyant tethered cylinder. Also, one of the attractive features of multipole expansion method for this type of problem is that the evaluation of hydrodynamic forces on a submerged circular cylinder in water is investigated (cf. Linton and McIver [6], Taylor and Hu [7], Sturova [8]).

The effect of the density changes of sea water are usually ignored in the early studies and assumption of uniform density was often used for the fluid. Recently, the effects of density inhomogeneity have attracted more and more attention. Wave propagation in a two-layer fluid with a free surface and an interface was first investigated by Stokes [9]. For such a two-layer fluid, It is known that for a particular frequency, there exist two modes 
of wave in a two-layer fluid, one propagating along the free surface and the other along the interface (cf. Art.231 of Lamb [10]). Linton and McIver [11] developed the general theory for two-dimensional wave motion in a two-layered fluid. The problem arose from modelling an underwater pipe bridge across Norwegian fjords consisting of a layer of fresh water on the top a deep layer of salt water. The three-dimensional wave scattering and radiation by a submerged sphere in a two-layer fluid is considered by Cadby and Linton (2000). Linton and Cadby [12] extended the work of Linton and McIver [13] to oblique scattering. Various aspects of wave motion have been analyzed in the two-layer fluids (cf. Manam and Sahoo [14], Chamberlian and Porter [15], Ten and Kashiwagi [16], Kashiwagi, Ten and Yasunaga [17] etc.).

Wave interaction with ice-cover (very large floating structure) is a front line area of research due to the practical utility in constructing floating offshore oil platforms, floating airports, floating pleasure cities, etc. The floating structure has elastic properties, and if it modelled as a thin elastic plate, then the boundary condition at the floating structure, when linearized, involves fifth order partial derivative of the potential function describing the irrotational motion in water in contrast the first order partial derivative in the free surface condition. This is also the case when the floating ice in an ice-covered ocean is modelled as a thin elastic floating plate. Wave propagation problems in the presence of floating elastic plate or floating sheet of ice are being investigated in recent times by mathematicians as well as ocean engineers due to a surge or scientific and oceanrelated industrial activities in polar region (cf. Fox and squire [18], Meylan and squire [19], Chakraborty [20], Squire [21], Das and Mandal [22,23], Das and Thakur [24], Thakur and Das [25], Das and sahu[26] and others). A more general class of problems in a twolayer fluid medium are the wave interaction with floating elastic plate or floating ice-sheet, which are of ocean engineering interest(cf. Das [27,28], Das and Mandal [29-31]). Also Das and Thakur [32] studied the wave scattering by a sphere in the two-layer fluid with an ice-cover. Recently, Sturova [8] also considered the problem of hydrodynamic loads acting on an oscillating cylinder submerged in a stratified fluid with ice-cover. Thakur and Das [25] investigated the wave scattering by submerged horizontal circular cylinder in water with an ice-cover to obtain the vertical and horizontal forces for infinite depth. Li et.all [33] considered the wave radiation and diffraction by a circular cylinder submerged below an ice-sheet with a crack. They used the multipole expansion method and the solution was obtained for a fluid of both finite and infinite depth. Recently, Sahu and Das [34] studied the hydrodynamic forces on a submerged horizontal circular cylinder in uniform finite depth water with ice-cover. This has motivated us to extend the problem in two layered fluid of which the upper layer is covered with thin ice-sheet. Here we obtain the horizontal and vertical forces in both the cylinder in lower and upper layer. It is observed that, when the cylinder is in the lower layer the vertical and horizontal forces are identical but they are $90^{\circ}$ out of phase but when the cylinder is in the upper layer they are different. The method of multipoles is used to reduce the problem to the solution of an infinite system of linear equations which are solved numerically by truncation. The time independent part of the vertical and horizontal forces are obtained and depicted graphically against the wave number for various values of flexural rigidity of the ice-cover. 


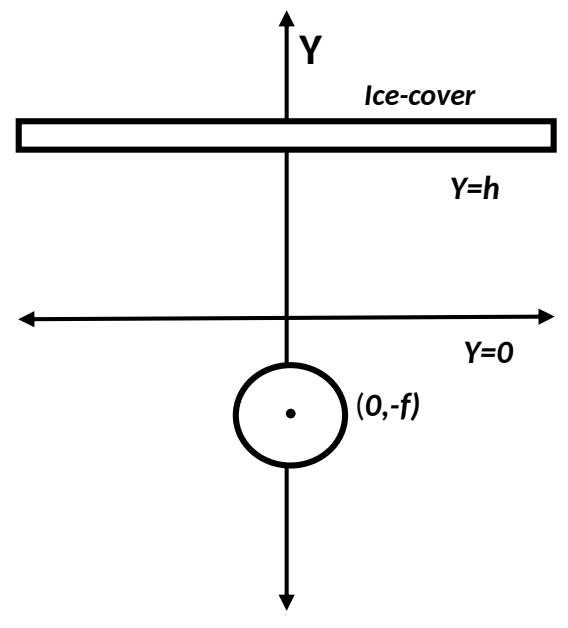

Fig1(a). Cylinder in lower layer

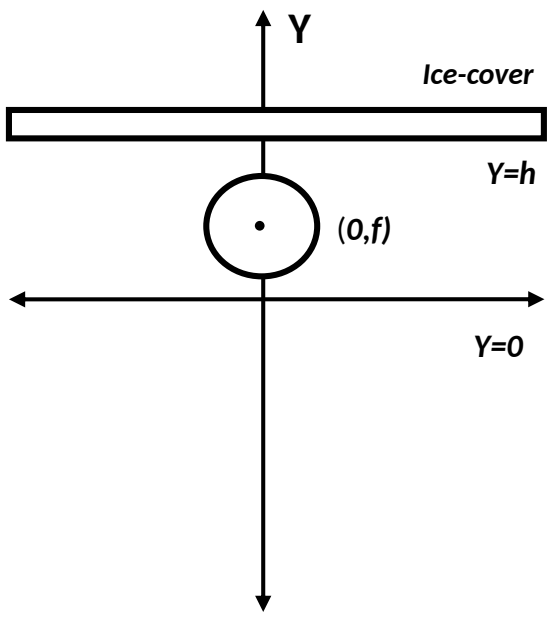

Fig 1(b). Cylinder in upper layer

\section{Mathematical Formulation}

We consider here the irrotational motion of two non-viscous incompressible fluids under the action of gravity, neglecting any effect due to surface tension at the interface of the two fluids, the upper being of finite depth $h$ and the lower layer is of infinite depth. The densities of upper and lower layers are $\rho_{1}$ and $\rho_{2}\left(\rho_{2}>\rho_{1}\right)$ respectively. A rectangular Cartesian co-ordinates are chosen such that $(x, z)$ - plane coincides with the undisturbed interface between the two fluids. The $y$ axis being measured vertically upwards and $y=0$ is the mean position of the interface and $y=h(>0)$ is the mean position of the ice-cover. (See fig. 1(a), 1(b)). Assuming the linear water wave theory, the time-dependent train of surface waves described by the potential function $\left\{\phi(x, y) e^{-i \sigma t}\right\}$, where $\phi(x, y)$ is the complex valued potential function and $\sigma$ denotes the angular frequency. The upper fluid, $0<y<h$ is referred to as region $I$, while the lower fluid, $y<0$ will be referred to as region $I I$ and the corresponding potential functions are $\phi^{I}$ and $\phi^{I I}$ respectively, which satisfy the Laplace equations (cf. Das and Mandal [30])

$$
\begin{gathered}
\nabla^{2} \phi^{I}=0, \quad \text { for } \quad 0<y<h, \\
\nabla^{2} \phi^{I I}=0, \quad \text { for } \quad y<0 .
\end{gathered}
$$

The linearized boundary conditions at the interface and the ice-cover are

$$
\begin{gathered}
\phi_{y}^{I}=\phi_{y}^{I I} \quad \text { on } y=0 \\
\rho\left(\phi_{y}^{I}-K \phi^{I}\right)=\phi_{y}^{I I}-K \phi^{I I} \quad \text { on } \quad y=0,
\end{gathered}
$$

where $\rho=\frac{\rho_{1}}{\rho_{2}}(<1)$ and

$$
\left(D \frac{\partial^{4}}{\partial x^{4}}+1-\epsilon K\right) \phi_{y}^{I}-K \phi^{I}=0, \text { on } y=h,
$$

where $K=\sigma^{2} / g, D=L /\left(\rho_{1} g\right), L$ being the flexural rigidity of the ice-cover, $\epsilon=\left(\rho_{0} / \rho_{1}\right) h_{0}$, $\rho_{0}$ is the density of the ice and $h_{0}$ is the small thickness of ice-cover. 
Also condition at large depth is

$$
\nabla \phi^{I I} \rightarrow 0 \quad \text { as } y \rightarrow-\infty .
$$

In a two layer fluid progressive waves have the form

$$
\phi^{I}=e^{ \pm i k x}\left[\left\{k\left(D k^{4}+1-\epsilon K\right)+K\right\} e^{k(y-h)}+\left\{k\left(D k^{4}+1-\epsilon K\right)-K\right\} e^{-k(y-h)}\right]
$$

and

$$
\phi^{I I}=e^{ \pm i k x} e^{k y}\left[\left\{k\left(D k^{4}+1-\epsilon K\right)+K\right\} e^{-k h}+\left\{k\left(D k^{4}+1-\epsilon K\right)-K\right\} e^{k h}\right],
$$

where $k$ satisfies the dispersion equation,

$$
\begin{aligned}
H(k)=K \rho & \left\{k\left(D k^{4}+1-\epsilon K\right) \cosh k h-K \sinh k h\right\}-\{k(1-\rho)-K\} \\
\times & \left\{k\left(D k^{4}+1-\epsilon K\right) \sinh k h-K \cosh k h\right\}=0 .
\end{aligned}
$$

The equation (2.9) has only two real positive roots $\lambda_{1}, \lambda_{2}\left(\lambda_{1}<\lambda_{2}\right)$ (cf. Das [27]).

When $k=\lambda_{j}(j=1,2)$, the progressive waves are thus of the form,

$$
\begin{array}{cl}
\phi^{I}(x, y)=e^{ \pm i \lambda_{j} x} g_{j}(y), & j=1,2, \\
\phi^{I I}(x, y)=e^{ \pm i \lambda_{j} x+\lambda_{j} y}, & j=1,2,
\end{array}
$$

where,

$$
\begin{aligned}
g_{j}(y)= & {\left[\left\{\lambda_{j}\left(D \lambda_{j}^{4}+1-\epsilon K\right)+K\right\} e^{\lambda_{j}(y-h)}+\left\{\lambda_{j}\left(D \lambda_{j}^{4}+1-\epsilon K\right)-K\right\} e^{-\lambda_{j}(y-h)}\right] } \\
& \times \frac{\left(\lambda_{j}(1-\rho)-K\right.}{K \rho\left\{\lambda_{j}\left(D \lambda_{j}^{4}+1-\epsilon K\right) \cosh \lambda_{j} h-K \sinh \lambda_{j} h\right\}}, \quad j=1,2 .
\end{aligned}
$$

Also $\phi^{I}(x, y)$ and $\phi^{I I}(x, y)$ behave an outgoing waves as $|x| \rightarrow \infty$.

\section{Cylinder in the lower layer}

Let a horizontal circular cylinder of radius $a$ have its axis $y=f(<0)$ and its generator runs parallel to $z$ axis. Polar co-ordinates $(r, \theta)$ are defined in the $(x, y)$ plane by

$$
x=r \sin \theta, \quad \text { and } \quad y=f-r \cos \theta .
$$

Let $\phi_{n}^{s}$ and $\phi_{n}^{a}$ denote the symmetric and anti-symmetric multipoles respectively which satisfy the boundary conditions. The multipoles are (cf. Das and Mandal[30])

$$
\begin{gathered}
\phi_{n}^{I s}=\frac{(-1)^{n}}{(n-1) !} \mathcal{f}_{0}^{\infty} k^{n-1}\left\{A(k) e^{k y}+B(k) e^{-k y}\right\} \cos k x d k, \\
\phi_{n}^{I I s}=\frac{\cos n \theta}{r^{n}}+\frac{(-1)^{n}}{(n-1) !} \int_{0}^{\infty} k^{n-1} C(k) e^{k y} \cos k x d k
\end{gathered}
$$




$$
\begin{gathered}
\phi_{n}^{I a}=\frac{(-1)^{n+1}}{(n-1) !} \mathcal{f}_{0}^{\infty} k^{n-1}\left\{A(k) e^{k y}+B(k) e^{-k y}\right\} \sin k x d k, \\
\phi_{n}^{I I a}=\frac{\sin n \theta}{r^{n}}+\frac{(-1)^{n+1}}{(n-1) !} \mathcal{f}_{0}^{\infty} k^{n-1} C(k) e^{k y} \sin k x d k
\end{gathered}
$$

where,

$$
\begin{gathered}
A(k)=K\left\{k\left(D k^{4}+1-\epsilon K\right)+K\right\} e^{k(f-h)} / H(k), \\
B(k)=K\left\{k\left(D k^{4}+1-\epsilon K\right)-K\right\} e^{k(f+h)} / H(k), \\
C(k)=\left[K \rho\left\{k\left(D k^{4}+1-\epsilon K\right) \cosh k h-K \sinh k h\right\}\right. \\
\left.-\{k(1-\rho)+K\}\left\{k\left(D k^{4}+1-\epsilon K\right) \sinh k h-K \cosh k h\right\}\right] e^{k f} / H(k),
\end{gathered}
$$

where the contour is intended below the poles $k=\lambda_{1}$ and $k=\lambda_{2}$, which satisfy the dispersion equation (2.9).

A power series expansion can be obtained from (3.3) and (3.5) as (cf. Das and Mandal [30]),

$$
\phi_{n}^{I I s}=\frac{\cos n \theta}{r^{n}}+\sum_{m=0}^{\infty} A_{n m} r^{m} \cos m \theta,
$$

and

$$
\phi_{n}^{I I a}=\frac{\sin n \theta}{r^{n}}+\sum_{m=0}^{\infty} A_{n m} r^{m} \sin m \theta
$$

where,

$$
A_{n m}=\frac{(-1)^{n+m}}{(n-1) ! m !} \int_{0}^{\infty} k^{n+m-1} C(k) e^{k f} d k .
$$

Incident wave due to a wave number $\lambda_{j}, j=1,2$ :

The incident wave potential $\phi_{i n c}^{I I}=e^{i \lambda_{j} x+\lambda_{j} y}$ has the expansion about $r=0$ as,

$$
\phi_{i n c}^{I I}=\sum_{m=0}^{\infty} \frac{(-1)^{m}}{m !} \lambda_{j}^{m} r^{m}(\cos m \theta-i \sin m \theta) e^{\lambda_{j} f} .
$$

For the symmetric and anti-symmetric part we write the multipole expansion as,

$$
\phi_{\lambda_{j}}^{I I s}=e^{\lambda_{j} y} \cos \lambda_{j} x+\sum_{n=1}^{\infty} a^{n} \alpha_{n} \phi_{n}^{I I s},
$$

and

$$
\phi_{\lambda_{j}}^{I I a}=i e^{\lambda_{j} y} \sin \lambda_{j} x+\sum_{n=1}^{\infty} a^{n} \beta_{n} \phi_{n}^{I I a},
$$

where, $\alpha_{n}$ and $\beta_{n}$ are unknown complex constants. Now, applying the body boundary condition $\frac{\partial \phi_{\lambda_{j}}^{I I}}{\partial r}=0$ on $r=a$ and using the orthogonal properties of trigonometric functions we get the two infinite systems of linear equations are,

$$
\alpha_{m}-\sum_{n=1}^{\infty} a^{n+m} A_{n m} \alpha_{n}=\frac{\left(-\lambda_{j} a\right)^{m}}{m !} e^{\lambda_{j} f}, \quad m=1,2, \cdots
$$




$$
\beta_{m}-\sum_{n=1}^{\infty} a^{n+m} A_{n m} \beta_{n}=-i \frac{\left(-\lambda_{j} a\right)^{m}}{m !} e^{\lambda_{j} f} . \quad m=1,2, \cdots
$$

These systems can be solved numerically by truncating the infinite series and solving an $N \times N$ system of equations. Here (3.15) and (3.16) are truncated upto five terms. For a submerged horizontal circular cylinder in infinite depth water and an incident wave of amplitude, the vertical exciting force is given by for both $\lambda_{1}$ and $\lambda_{2}$,

$$
X_{V}=\rho g A \int_{-\pi}^{\pi} \phi_{n}^{I I s}(a, \theta) \cos \theta a d \theta
$$

Using orthogonality of the trigonometric functions we have, (cf. Thakur and Das [25])

$$
X_{V}=2 \pi \rho g A a \alpha_{1}
$$

Similarly, the horizontal exciting force is given by,

$$
X_{H}=2 \pi \rho g A a \beta_{1} .
$$

(3.18) Comparing (3.15) and (3.16) we have, $\beta_{n}=-i \alpha_{n}$. Then,

$$
X_{H}=-2 i \pi \rho g A a \alpha_{1} .
$$

Here, it is seen that the amplitudes of these oscillatory forces are identical, but they are $90^{0}$ out of phase.

\section{Numerical Results}

Figures 2,3 show the non-dimensional vertical exciting forces on the horizontal cylinder in lower layer due to an incident wave of wave number $\lambda_{1}$ and 4,5 show that the vertical forces in lower layer due to an incident wave number $\lambda_{2}$. Fig.2 Shows vertical forces against $K a$ for $h / a=2, f / a=-3, \rho=.95$ and for different values of $D / a^{4}$. It is observed that, first the vertical forces increase as $K a$ increases from low to moderate values, then decrease as $K a$ further increase for any value of $D / a^{4}$, but forces increase $D / a^{4}$ increases with increasing of $K a$. When $D / a^{4}=.001, \epsilon / a=.001$ the curve coincides to the curve for the case of two-layer fluid with free surface. In Fig.3 the vertical forces is plotted against $K a$, for $h / a=2, D / a^{4}=1, \epsilon / a=.01, \rho=.95$ and for different values of $f / a$. These figures show that, the vertical forces first increase then after attending its max values, decrease very slowly. In Fig. $4,\left|X_{V}\right|$ is plotted against $K a$ for $f / a=-2, h / a=2$, $\rho=.95$ and for different values of $D / a^{4}$. From the figures it is observed that,vertical forces first increase from low to moderate values then decrease as $K a$ increases. When $D / a^{4}=.001, \epsilon / a=.001$ the vertical exciting forces are the same as in case of two-layer fluid with free surface. In Fig5., $\left|X_{V}\right|$ is plotted against $K a$ for $D / a^{4}=1, h / a=2$, $\rho=.95, \epsilon / a=.01$ and for various values of $K a$. First $\left|X_{V}\right|$ increases then decreases as $K a$ further increases.

The horizontal forces for the cases of incident waves $\lambda_{1}$ and $\lambda_{2}$ are not shown here. The magnitude of the horizontal forces are equal to the magnitude of the vertical forces for both the incident wave of wave numbers and display the same qualitative effects. Also it 
is noted that when the cylinder is closed to the interface $(f / a=-2)$ the vertical forces are greater as more wave energy are generated at the interface than on the larger depth of the cylinder from the interface.

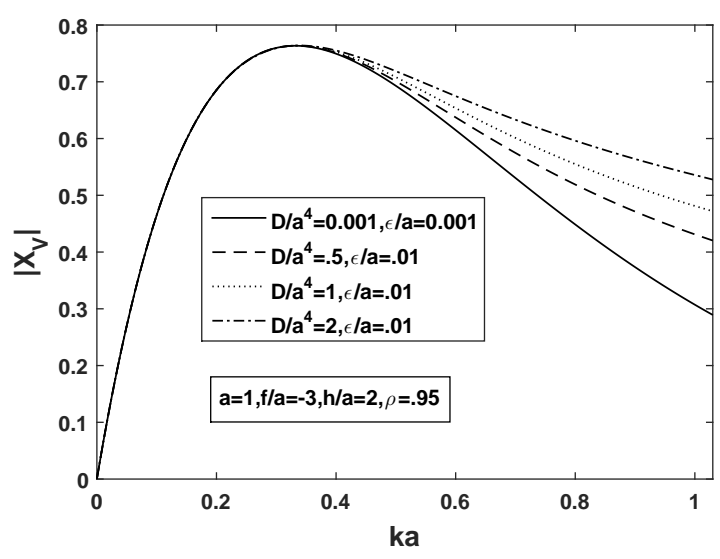

Fig 2. Vertical forces due to an incident wave of wave number $\lambda_{1}$ in lower layer

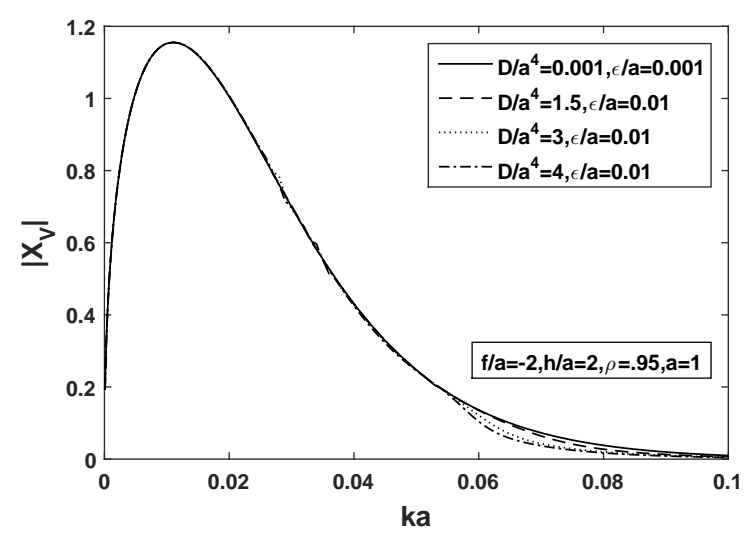

Fig 4. Vertical forces due to an incident wave of wave number $\lambda_{2}$ in lower layer

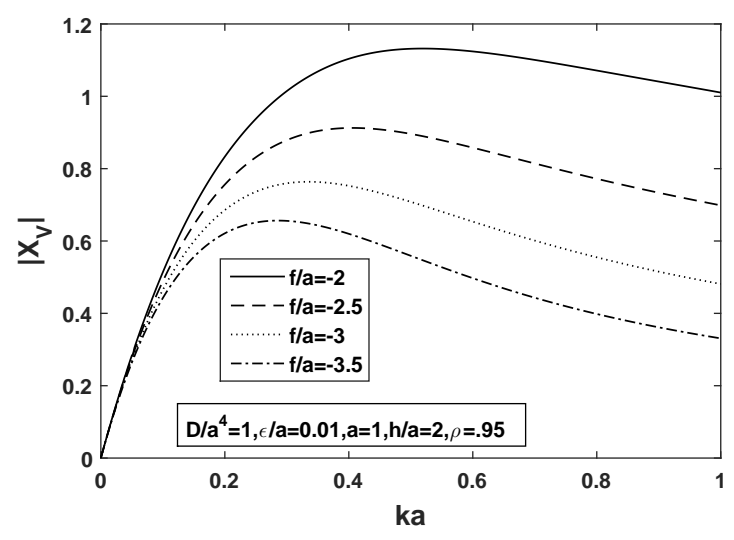

Fig 3. Vertical forces due to an incident wave of wave number $\lambda_{1}$ in lower layer

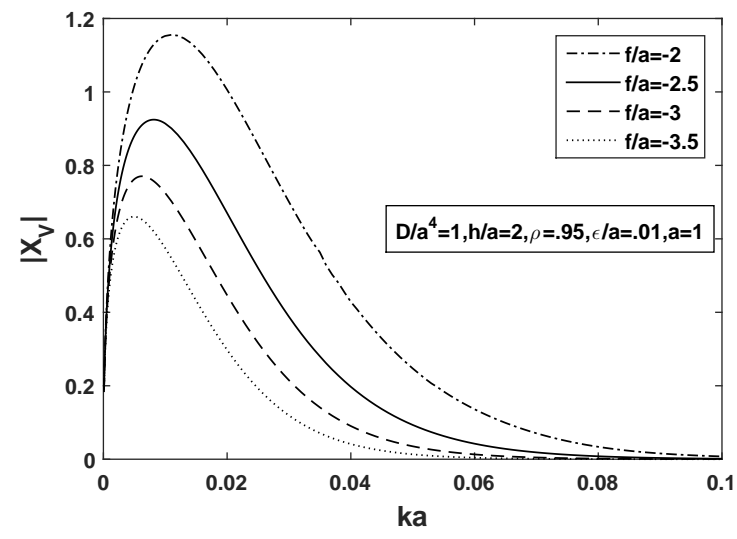

Fig 5. Vertical forces due to an incident wave of wave number $\lambda_{2}$ in lower layer

\section{Cylinder in the upper layer}

Let a horizontal circular cylinder of radius a have its axis $y=f(>0)$ and its generator runs parallel to $z$ axis $(f>a)$. Polar co-ordinates $(r, \theta)$ are defined in the $(x, y)$ plane by $x=r \sin \theta$, and $y=f-r \cos \theta$. The potential functions are (cf. Das and Mandal [30])

$$
\begin{gathered}
\phi_{n}^{I s}=\frac{\cos n \theta}{r^{n}}+\frac{1}{(n-1) !} \mathcal{f}_{0}^{\infty} k^{n-1}\left\{A_{n}^{(0)}(k) e^{k y}+B_{n}^{(0)}(k) e^{-k y}\right\} \cos k x d k, \\
\phi_{n}^{I I s}=\frac{1}{(n-1) !} f_{0}^{\infty} k^{n-1} C_{n}^{(0)}(k) e^{k y} \cos k x d k,
\end{gathered}
$$




$$
\begin{gathered}
\phi_{n}^{I a}=\frac{\sin n \theta}{r^{n}}+\frac{1}{(n-1) !} \mathcal{f}_{0}^{\infty} k^{n-1}\left\{A_{n}^{(1)}(k) e^{k y}+B_{n}^{(1)}(k) e^{-k y}\right\} \sin k x d k, \\
\phi_{n}^{I I a}=\frac{1}{(n-1) !} \int_{0}^{\infty} k^{n-1} C_{n}^{(1)}(k) e^{k y} \sin k x d k,
\end{gathered}
$$

where,

$$
\begin{gathered}
A_{n}^{(j)}(k)=\frac{1}{2}\left\{k\left(D k^{4}+1-\epsilon K\right)+K\right\} e^{-k h} \\
\times \frac{(-1)^{n+j+1}((1-\rho) k-(1+\rho) K) e^{k f}-(1-\rho)(k-K) e^{-k f}}{H(k)}, \\
B_{n}^{(j)}(k)=\frac{1}{2} \frac{(-1)^{n+j+1}\left\{k\left(D k^{4}+1-\epsilon K\right)+K\right\}(1-\rho)(k-K) e^{k(f-h)}}{H(k)} \\
-\frac{1}{2} \frac{(1-\rho)(k-K)\left\{k\left(D k^{4}+1-\epsilon K\right)-K\right\} e^{-k(f-h)}}{H(k)}, \\
C_{n}^{(j)}(k)=-\rho K \\
\times \frac{\left[(-1)^{n+j+1}\left\{k\left(D k^{4}+1-\epsilon K\right)+K\right\} e^{k(f-h)}-\left\{k\left(D k^{4}+1-\epsilon K\right)-K\right\} e^{-k(f-h)}\right]}{H(k)},
\end{gathered}
$$

where $j=0,1$, the contour is intended below the poles $k=\lambda_{1}$ and $k=\lambda_{2}$, in the complex $k$ plane.

A power series expansion can be obtained from (4.1) and (4.3) as (cf. Das and Mandal [30]),

$$
\phi_{n}^{I s}=\frac{\cos n \theta}{r^{n}}+\sum_{m=0}^{\infty} E_{n m}^{s} r^{m} \cos m \theta
$$

and

$$
\phi_{n}^{I a}=\frac{\sin n \theta}{r^{n}}+\sum_{m=0}^{\infty} E_{n m}^{a} r^{m} \sin m \theta,
$$

where,

$$
\begin{gathered}
E_{n m}^{s}=\frac{1}{(n-1) ! m !} \mathcal{f}_{0}^{\infty} k^{n+m-1}\left((-1)^{m} A_{n}^{(0)}(k) e^{k f}+B_{n}^{(0)}(k) e^{-k f}\right) d k, \\
E_{n m}^{a}=\frac{1}{(n-1) ! m !} \mathcal{T}_{0}^{\infty} k^{n+m-1}\left((-1)^{m+1} A_{n}^{(1)}(k) e^{k f}+B_{n}^{(1)}(k) e^{-k f}\right) d k .
\end{gathered}
$$

Incident wave due to a wave number $\lambda_{j}, j=1,2$ :

The incident wave potential $\phi_{i n c}^{I}=e^{i \lambda_{j} x} g_{j}(y)$ has the expansion about $r=0$ as,

$$
\begin{gathered}
\phi_{\text {inc }}^{I}=\sum_{m=0}^{\infty} \frac{\left(\lambda_{j} r\right)^{m}}{m !}\left[\left\{(-1)^{m} M_{1}^{\lambda_{j}} e^{-\lambda_{j}(h-f)}+M_{2}^{\lambda_{j}} e^{\lambda_{j}(h-f)}\right\} \cos m \theta+\right. \\
\left.i\left\{(-1)^{m+1} M_{1}^{\lambda_{j}} e^{-\lambda_{j}(h-f)}+M_{2}^{\lambda_{j}} e^{\lambda_{j}(h-f)}\right\} \sin m \theta\right]
\end{gathered}
$$


where,

$$
M_{1,2}^{\lambda_{j}}=\frac{\left\{(1-\rho) \lambda_{j}-K\right\}\left\{\lambda_{j}\left(D \lambda_{j}^{4}+1-\epsilon K\right) \pm K\right\}}{K \rho\left\{\lambda_{j}\left(D \lambda_{j}^{4}+1-\epsilon K\right) \cosh \lambda_{j} h-K \sinh \lambda_{j} h\right\}} .
$$

The velocity potential $\phi_{\lambda_{j}}^{I s}$ and $\phi_{\lambda_{j}}^{I a}$ is expanded as in (3.13) and (3.14) $\left(\phi^{I I}\right.$ replace by $\left.\phi^{I}\right)$. Now applying body boundary condition $\frac{\partial \phi_{\lambda_{j}}^{I}}{\partial r}=0$ on $r=a$ and using the orthogonal properties of trigonometric functions we get the two infinite systems of linear equations are,

$$
\begin{gathered}
\alpha_{m}-\sum_{n=1}^{\infty} a^{n+m} E_{n m}^{s} \alpha_{n}=\frac{\left(\lambda_{j} a\right)^{m}}{m !}\left[(-1)^{m} M_{1}^{\lambda_{j}} e^{-\lambda_{j}(h-f)}+M_{2}^{\lambda_{j}} e^{\lambda_{j}(h-f)}\right], m=1,2, \cdots \\
\beta_{m}-\sum_{n=1}^{\infty} a^{n+m} E_{n m}^{a} \beta_{n}=i \frac{\left(\lambda_{j} a\right)^{m}}{m !}\left[(-1)^{m+1} M_{1}^{\lambda_{j}} e^{-\lambda_{j}(h-f)}+M_{2}^{\lambda_{j}} e^{\lambda_{j}(h-f)}\right] . m=1,2, \cdots
\end{gathered}
$$

Again this system can be solved by truncation. For a submerged horizontal circular cylinder in finite depth water and an incident wave of amplitude $A$, the vertical exciting force is given by,

$$
X_{V}=\rho g A \int_{-\pi}^{\pi} \phi_{n}^{I s}(a, \theta) \cos \theta a d \theta .
$$

Using orthogonality of the trigonometric functions we have,

$$
X_{V}=2 \pi \rho g A a \alpha_{1} .
$$

Similarly, the horizontal exciting force is given by,

$$
X_{H}=2 \pi \rho g A a \beta_{1} \text {. }
$$

\section{Numerical Results}

Figures 6, 7 show the non-dimensional vertical forces and 8, 9 show the non-dimensional horizontal forces on the cylinder in upper layer due to an incident wave of wave number $\lambda_{1}$ and Fig.10, 11 for vertical forces, Fig.12, 13 for horizontal forces corresponding to $\lambda_{2}$. From Fig6. it is observed that for the vertical forces for different values of $D / a^{4}$ first increase with decreases of $D / a^{4}$ and after a moderate values of the curve decrease with decreases of $D / a^{4}$. In Fig.10, We see that the vertical forces due to an incident wave of wave number $\lambda_{2}$ have similar features but smaller than the case of incident wave of wave number $\lambda_{1}$. In Fig.7 and 11 it is seen that the vertical forces for both the cases increase when the depth of centre of the submerged circular cylinder decreases i.e. for nearer to the ice-cover surface. Also it is observed that the forces are oscillatory in nature due to an interaction of the incident wave between the boundary of the circular cylinder, ice-cover surface and interface between two-layer. 


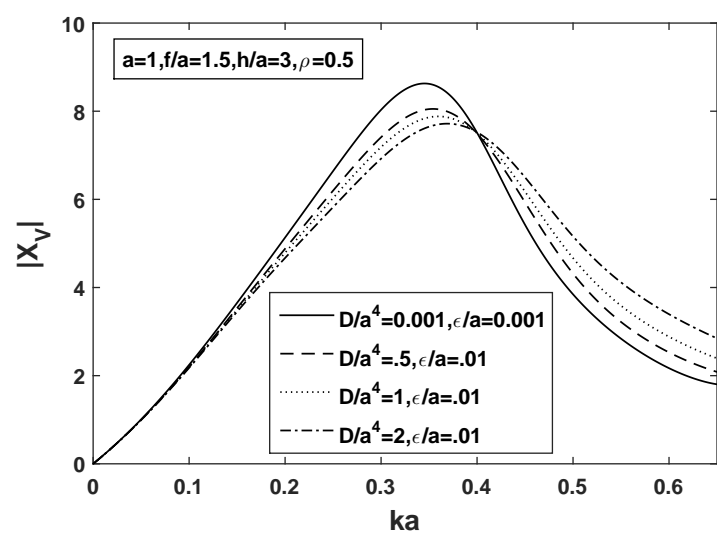

Fig 6. Vertical forces due to an incident wave of wave number $\lambda_{1}$ in upper layer

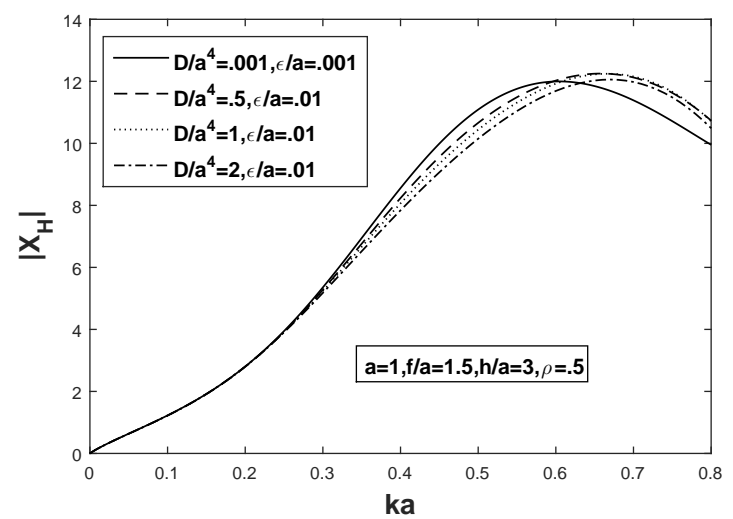

Fig 8. Horizontal forces due to an incident wave of wave number $\lambda_{1}$ in upper layer

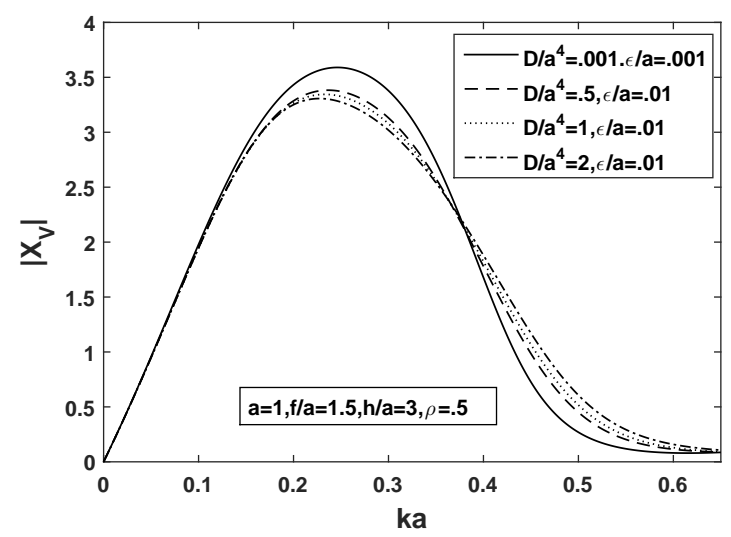

Fig 10. Vertical forces due to an incident wave of wave number $\lambda_{2}$ in upper layer

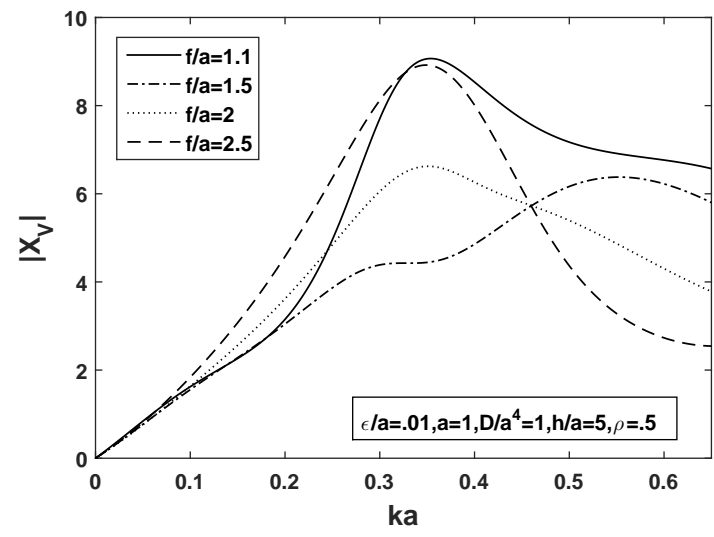

Fig 7. Vertical forces due to an incident wave of wave number $\lambda_{1}$ in upper layer

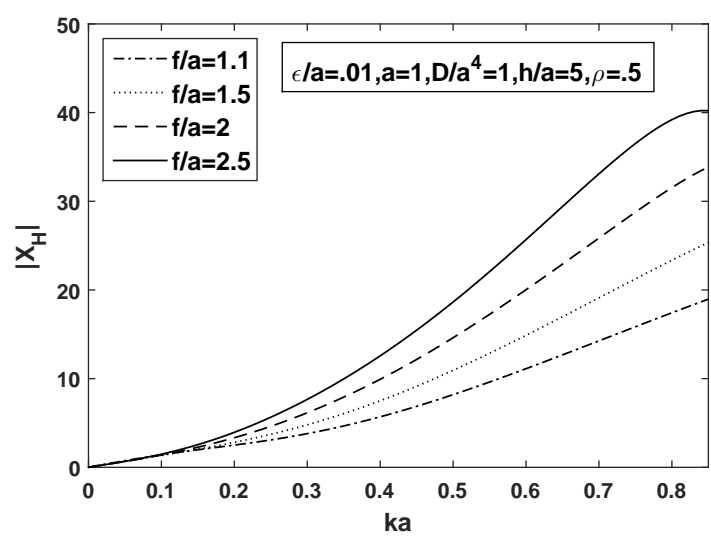

Fig 9. Horizontal forces due to an incident wave of wave number $\lambda_{1}$ in upper layer

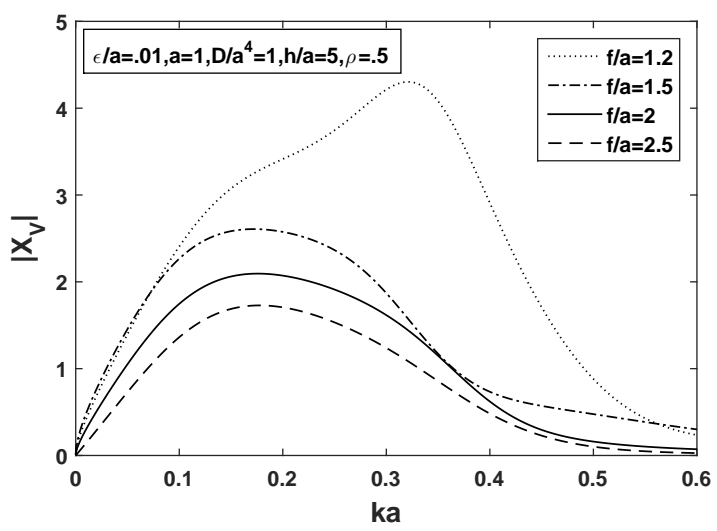

Fig 11. Vertical forces due to an incident wave of wave number $\lambda_{2}$ in upper layer 


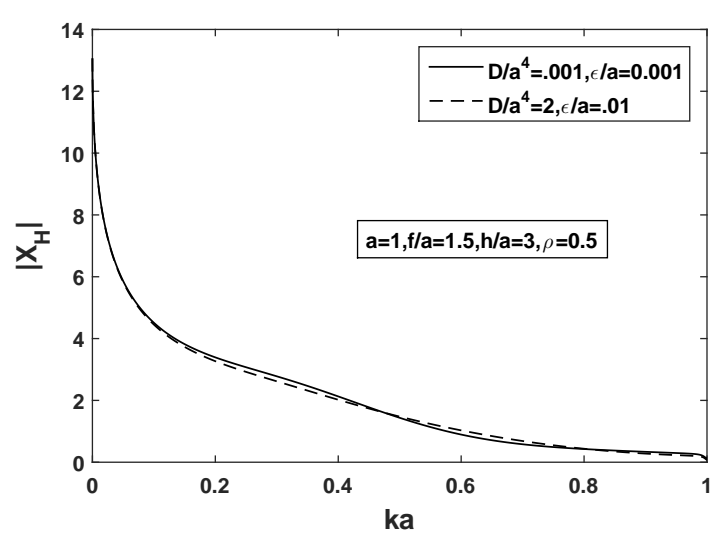

Fig 12. Horizontal forces due to an incident wave of wave number $\lambda_{2}$ in upper layer

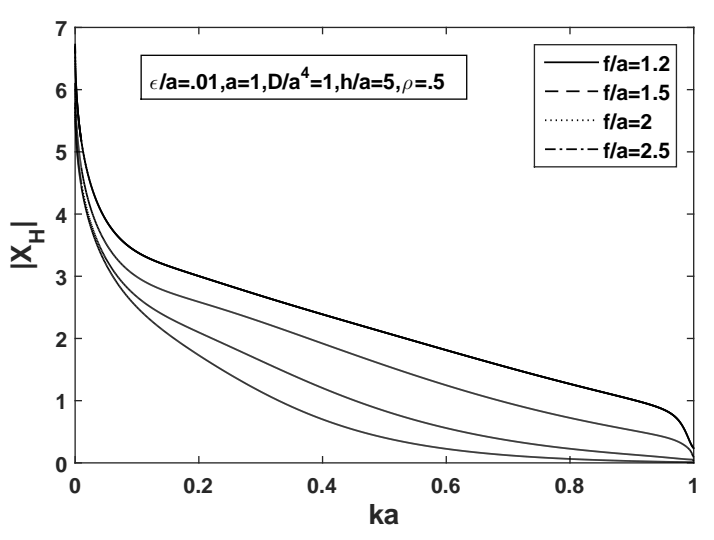

Fig 13. Horizontal forces due to an incident wave of wave number $\lambda_{2}$ in upper layer

Fig. 8 and 12 show the horizontal forces for different values of $D / a^{4}$ due to an incident wave of wave number $\lambda_{1}$ and $\lambda_{2}$ respectively. These show the effect of the flexural rigidity of the ice-sheet and also observed that when $D / a^{4}=.001, \epsilon / a=.001$, the forces are similar to the case of free surface two-layer fluid. Fig.9 and 13 show the horizontal forces for different values of $f / a$. For both the cases, it is observed that the forces increase with the increase of $f / a$. Also it is noted that from the figures the forces in the lower layer are smaller than that for upper layer for any value of $D / a^{4}$.

\section{Conclusion}

The problem of hydrodynamic forces on the submerged circular cylinder is extended here for a two-layer fluid. The lower is of infinite depth and the upper layer is of finite depth with an ice-cover surface, modelled as a thin elastic plate. Numerical results for the vertical and the horizontal forces on the cylinder are obtained. The method of multipoles has been shown to be an extremely powerful method for solving this problem involving submerged circular cylinder. The vertical forces on the cylinder in lower layer and also vertical and horizontal forces on the cylinder in upper layer are depicted graphically against the wave number in a number of figures. When $D / a^{4}=0.001$ and $\epsilon / a=0.001$, the forces are coincided with the curves for the case of two-layer fluid with free surface.

\section{References}

[1] Dean W. R., On the reflection of surface waves by a submerged circular cylinder, Proc. Camb. Phils. Soc., 44 (1948)957-983.

[2] Ursel, F., Surface wave on deep water in the presence of submerged circular cylinder Iand II, Proc. Camb. Phil. Soc., 46(1950)141-155.

[3] Jackson J. D., Classical Electrodynamics, Wiley Eastern, (1978).

[4] Morse P.H. and Feshbach H., Method of Theoretical Physics, McGraw-Hill, Lond., (1953). 
[5] Evans D. V. and Linton C. M., Active devices for the radiation of wave intensity, Appl. Ocean Res., 11 (1989) 26-32.

[6] Linton C. M. and McIver P., Handbook of Mathematical Techniques for Wave/Structure Interactions, Chapman and Hall/CRC, (2001).

[7] Taylor e.R. and Hu C. S., Multipole expansions for wave diffraction and radiation in deepwater, Ocean Engng., 18(1991) 191-224.

[8] Sturova I. V.,Hydrodynamic loads acting on an oscillating cylinder submerged in a stratified fluid with ice cover, J.Appl.Mech.Tech.Phys., 52(2011) 415-426.

[9] Stokes G.G., On the theory of Oscillatory Waves. Trans. Camb. Phill. Soc. 8(1847)441-445, Reprinted in Maths. Phys. papers, Cambridge University Press. $1(1847) 314-326$.

[10] Lamb H., Hydrodynamics. Cambridge University Press. (1932).

[11] Linton C.M. and McIver M., The interaction of waves with horizontal cylinders in two-layer fluids. J. Fluid Mech. 304(1995)213-229.

[12] Cadby J.R. and Linton C.M., Three-dimensional water wave scattering in two-layers fluids. J. Fluid Mech. 423(2000)155-173.

[13] Linton C.M. and Cadby J.R., Scattering of oblique waves in a two-layer fluid. J. Fluid Mech. 461(2002)343-364.

[14] Manam S.R. and Sahoo T., Waves past porous structures in a two-layer fluid. J. Eng. Math. 52(2005) 355-377.

[15] Chamberlian P.G. and Porter D., Wave scattering in a two-layer fluid of varying depth. J. Fluid Mech. 524 (2005) 207-228.

[16] Ten I. and Kashiwagi M., Hydrodynamics of a body floating in a two- layer fluid of finite depth. part 1. Radiation problem. J. Mar. Sci. Tech. 9(2004) 127-141.

[17] Kashiwagi M., Ten I. and Yesunaga M., Hydrodynamics of a body floating in a twolayer fluid of finite depth, part 2. Diffraction problem and wave incident motion. J. Mar. Sci. Tech. 11(2006) 150-164.

[18] Fox C. and Squire V.A., On the oblique reflexion and transmission of ocean waves from shore fast sea ice, Philos. Trans. R. Soc. Lond. A347( 1994)185-218.

[19] Meylan M. and Squire V.A., The response of ice floes to ocean waves, J. Geophys. Res. 99c1(1994)891-900.

[20] Chakrabarti A., On the solution of the problem of scattering of surface water waves of the edge of an ice-cover, Proc. R. Soc. Lond. A456(2000)1087-1099.

[21] Squire V.A. ,Of ocean waves and sea-ice revisited. Cold. Reg. Sci. Technol., 49( 2007)110-133. 
[22] Das D.and Mandal B.N. ,Oblique wave scattering by a circular cylinder submerged beneath an ice-cover. Int. J. Eng. Sci. 44( 2006)166-179.

[23] Das D. and Mandal B.N., Water wave radiation by a sphere submerged in water with an ice-cover. Arch. Appl. Mech. 78(2008)649-461.

[24] Das, D. and Thakur, N., Water wave scattering by a sphere submerged in uniform finite depth water with an ice-cover, J. Marine Structures 30(2013) 63-73.

[25]Thakur, N. and Das, D., Hydrodynamic Forces on a Submerged horizontal circular cylinder in water with an ice-cover, Iranian J. Sci. Tech. Trans. A: Sci.41(2017) 837-842.

[26] Das D. and sahu M., Wave Radiation by a horizontal circular cylinder submerged in deep water with ice-cover, J. Ocean Engng. Sci. 4(2019) 49-54.

[27] Das D., Solution of the dispersion equation for internal waves in two-layer fluid with an ice-cover. Bull. Cal. Math. Soc. 100(2)(2008)165-176.

[28] Das D., Construction of wave-free potentials and multipoles in a two-layer fluid having free surface boundary condition with higher-order derivatives. J. Mar. aci. appl. 14(2015)271-282.

[29] Das D. and Mandal B.N., Oblique wave scattering by a circular cylinder in two-layer fluid with an ice-cover. 21st IWWWFB, Loughborough University, UK, 2-5 April (2006) 29-32.

[30] Das, D. and Mandal, B. N., Wave scattering by a horizontal circular cylinder in a two-layer fluid with an ice-cover, Int. J. Engng. Sci. 45(2007) 842-872.

[31] Das, D. and Mandal, B. N., Wave radiation by a sphere submerged in a two-layer ocean with an ice-cover, Applied Ocean Research 32(2010) 358-366.

[32] Das, D. and Thakur, N., Wave scattering by a sphere submerged in a two-layer fluid with an ice-cover, International J. Appl. Math. Engng. Sci. 8(2014) 45-63.

[33] Li Z. F., Wu G. X and Ji C. Y., Wave diffraction by a circular crack in an ice sheet floating on water of finite depth, Phys. Fluid. 30(2018)682-712.

[34] Sahu M. and Das D., Hydrodynamic forces on a submerged horizontal circular cylinder in uniform finite depth ive covered water, Int. J. Appl. Mech. Engng. Sci. 25(2020)219-227. 